T. Kaneyama

Nagoya Math. J.

Vol. 57 (1975), 65-86

\title{
ON EQUIVARIANT VECTOR BUNDLES ON AN ALMOST HOMOGENEOUS VARIETY
}

\author{
TAMAFUMI KANEYAMA
}

\section{§ 1. Introduction}

Let $k$ be an algebraically closed field of arbitrary characteristic. Let $T$ be an $n$-dimensional algebraic torus, i.e. $T=G_{m} \times \cdots \times G_{m}(n$ times), where $G_{m}=\operatorname{Spec}\left(k\left[t, t^{-1}\right]\right)$ is the multiplicative group.

An almost homogeneous variety under an action of $T$ is an algebraic variety $X$ over $k$ endowed with an action of $T$ and which has a dense orbit. Normal effective almost homogeneous varieties under torus action have been classified in $[2,4]$. We review the results briefly in $\S 2$.

Let $E$ be a vector bundle on an almost homogeneous variety $X$ with an action of $T$. For every $k$-rational point $t$ in $T$, the action of $t$ on $X$ is denoted by $t: X \rightarrow X(x \mapsto t x)$. We say that $E$ is equivariant if there exists an isomorphism $\phi_{t}: t^{*} E \stackrel{\sim}{\longrightarrow} E$ for every $k$-rational point $t$ of $T$. Furthermore we say that an equivariant vector bundle $E$ is $T$ linearized if, for every pair of $k$-rational points $t, t^{\prime}$ of $T, \phi_{t t^{\prime}}=\phi_{t^{\prime}} \cdot t^{\prime *} \phi_{t}$ holds where

$$
\phi_{t^{\prime}} \cdot t^{\prime *} \phi_{t}:\left(t t^{\prime}\right) * E=t^{*} t^{*} E \stackrel{t^{*} \phi_{t}}{\longrightarrow} t^{*} E \stackrel{\phi_{t^{\prime}}}{\longrightarrow} E
$$

In this paper we study equivariant vector bundles on a smooth complete almost homogeneous variety. In $\S 3$ we show that an equivariant vector bundle $E$ on $X$ always has a $T$-linearization. Thus we study $T$-linearized vector bundles on $X$. Let $\left\{U_{i}\right\}$ be a covering of $X$ by $T$-stable affine open sets. We show that the restriction of $E$ to $U_{i}$ has a semi-invariant base with respect to the action of $T$, where a section $u \in E\left(U_{i}\right)$ is semiinvariant if for some character $\xi$

$$
\phi_{t}(u)=e(\xi)(t) u
$$

Received July 26, 1974. 
holds for every $k$-rational point $t$ in $T . \quad e(\xi)(t)$ is the value at $t$ of the function $\xi: T \rightarrow G_{m}$. Then in $\S 4$ we describe a $T$-linearized vector bundle $E$ on $X$ in terms of these semi-invariant, i.e.

Theorem 4.2 Let $(X, T)$ be a smooth complete almost homogeneous variety defined by a cone complex $(\Gamma, \mathscr{C})$. The set of $T$-linearized vector bundles of rank $r$ up to $T$-isomorphism corresponds bijectively to the set of $(m, P)$ up to equivalence. A detailed description of $(m, P)$ is in $\S 4$.

Finally, we give examples of equivariant vector bundle of rank 2 on $\boldsymbol{P}^{2}$ which are indecomposable.

The auther wishes to thank Professor T.Oda for his advise during the preparation of this paper.

\section{§ 2. Almost homogeneous varieties}

Let $(X, T)$ be an $n$-dimensional almost homogeneous variety where $T$ is an $n$-dimensional algebraic torus. Let $\Xi=\Xi(T)=\operatorname{Hom}_{k-g r}\left(T, G_{m}\right)$ be the additive group of characters of $T . \quad \Xi$ is a free $Z$-module of rank $n=\operatorname{dim}(T)$. Let the exponential map $e: \Xi \rightarrow k(T)^{*}$ be the homomorphism which sends $\xi$ in $\Xi$ to the corresponding rational function $e(\xi)$ on $T$. Let $\Gamma=\operatorname{Hom}_{k-g r}\left(G_{m}, T\right)$ be the additive group of one-parameter subgroup of $T$. $\Gamma$ is a free $Z$-module of $\operatorname{rank} n=\operatorname{dim}(T)$ and is the dual $Z$ module of $E$.

We call a non-empty subset $C$ of $\Gamma_{\boldsymbol{Q}}=\Gamma \otimes_{Z} \boldsymbol{Q}$ a strongly convex polyhedral cone with apex at 0 , or simply a cone, if $C \cap(-C)=\{0\}$ and if there exists a finite subset $\left\{\phi_{1}, \cdots, \phi_{m}\right\}$ of $\Gamma$ such that $C=\boldsymbol{Q}_{0} \phi_{1}+\cdots$

$+\boldsymbol{Q}_{0} \phi_{m}$ where $\boldsymbol{Q}_{0}$ denotes the set of non-negative rational numbers. If $\left\{\phi_{1}, \cdots, \phi_{m}\right\}$ is irredundant and each $\phi_{i}$ is primitive, i.e. is an integral multiple of no element of $\Gamma$, we call $\phi_{1}, \cdots, \phi_{m}$ verticial elements of the cone $C$. Let the dimension of $C$ be the dimension of the $Q$-vector space $C+(-C)$. A non-empty subset $C^{\prime}$ of a cone $C$ is called a facial cone of $C$ if there exists an element $\xi$ of $\Xi$ such that $\phi(\xi) \geqq 0$ for all $\phi$ in $C$ and that $C^{\prime}=\{\phi \in C \mid \phi(\xi)=0\}$.

A cone complex $(\Gamma, \mathscr{C})$, or simyly $\mathscr{C}$, in $\Gamma_{\boldsymbol{Q}}$ is a finite collection of cones of $\Gamma_{\boldsymbol{Q}}$ with properties:

(i) if $C^{\prime}$ is a facial cone of $C$ in $\mathscr{C}$ then $C^{\prime}$ is in $\mathscr{C}$,

(ii) if $C$ and $C^{\prime}$ are in $\mathscr{C}$ then the intersection $C \cap C^{\prime}$ is a facial cone of $C$ as well as of $C^{\prime}$. 
THEOREM 2.1. The category of normal effective almost homogeneous varieties under torus action and equivariant morphisms is equivalent to the category of pairs $(\Gamma, \mathscr{C})$ consisting of a free $Z$-module $\Gamma$ of finite rank and a cone complex $\mathscr{C}$ in $\Gamma_{\boldsymbol{Q}}$ and maps which are defined as follows:

A map from a pair $(\Gamma, \mathscr{C})$ to another pair $\left(\Gamma^{\prime}, \mathscr{C}^{\prime}\right)$ is a homomorphism of finite cokernel from $\Gamma$ to $\Gamma^{\prime}$ whose scalar extension to $\boldsymbol{Q}$ sends any cone of $\mathscr{C}$ into some cone of $\mathscr{C}^{\prime}$.

Moreover, $X$ is complete if and only if the associated pair satisfies $\Gamma_{Q}=\cup_{c \in \mathscr{Q}} C . \quad X$ is smooth if and only if every cone (resp. every maximal cone) in $\mathscr{C}$ is regular, i.e. the set of its varticial elements can be extended to a $Z$-base of $\Gamma$.

See [4].

Proposition 2.2. Let $(X, T)$ be a smooth almost homogeneous variety and let $x$ in $X$ be a T-fixed closed point. Let $V$ be the union of T-orbits whose closure contains $x$. Then there exists a base $\left\{\alpha_{1}, \cdots, \alpha_{n}\right\}$ of $\Xi$ such that $V$ is T-equivariantly isomorphic to the affine space $\operatorname{Spec}\left(k\left[e\left(\alpha_{1}\right), \cdots\right.\right.$, $\left.\left.e\left(\alpha_{n}\right)\right]\right)$ which has the canonical diagonal T-action $e(\xi) \mapsto e(\xi) \otimes e(\xi)$.

See [4].

\section{§ 3. Equivariant vector bundles}

From now on $(X, T)$ is a complete smooth almost homogeneous variety unless otherwise stated. Hence $X$ is covered by $T$-stable open sets isomorphic to the $n$-dimensional affine space with a diagonal action of $T$ by proposition 2.2. For every $k$-rational point $t$ of $T$, the action on $X$ of $t$ is denoted by $t: X \rightarrow X$ sending $x$ to $t x$.

DEFINITION 3.1. An equivariant vector bundle $E$ on $(X, T)$ is the vector bundle on $X$ such that, for every $k$-rational point $t$ in $T$, there exists an isomorphism $\phi_{t}: t^{*} E \stackrel{\sim}{\longrightarrow} E$.

DEFinition 3.2. An equivariant vector bundle $\left(E, \phi_{t}\right)$ is called $T$ linearized if, for every pair of $k$-rational points $t, t^{\prime}$ of $T, \phi_{t t^{\prime}}=\phi_{t^{\prime}} \cdot t^{\prime *} \phi_{t}$ holds where

$$
\phi_{t^{\prime}} \cdot t^{* *} \phi_{t}:\left(t t^{\prime}\right) * E=t^{* *} t^{*} E \stackrel{t^{* *} \phi_{t}}{\longrightarrow} t^{*} E \stackrel{\phi_{t^{\prime}}}{\longrightarrow} E
$$


Let $E$ be an equivariant vector bundle on $(X, T)$. Let $G(E)$ be the set of pairs $\left(t, \phi_{t}\right)$ where $t$ is a $k$-rational point of $T$ and $\phi_{t}$ is an isomorphism $\phi_{t}: t^{*} E \rightarrow E . \quad G(E)$ is an algebraic group whose multiplication is given by.

$$
(t, \phi)\left(t^{\prime}, \phi^{\prime}\right)=\left(t t^{\prime}, \phi^{\prime} \cdot t^{\prime *} \phi\right)
$$

Then $G(E)$ is an extension of $T$ by the bundle automorphism group Aut $(E)$

$$
0 \longrightarrow \operatorname{Aut}(E) \stackrel{j}{\longrightarrow} G(E) \stackrel{p}{\longrightarrow} T \longrightarrow 0
$$

$j$ sends $\phi$ to $(1, \phi)$ and $p$ sends $(t, \phi)$ to $t$. Note that a $T$-linearization for $E$ corresponds to giving a group section $s: T \rightarrow G(E)$.

Proposition 3.3. The above exact sequence has a group section $s: T \rightarrow G(E)$. Hence equivariant vector bundle can always be T-linearized.

Proof. Since $T$ and Aut $(E)$ are linear algebraic group, so is $G(E)$. Let $T^{\prime}$ be a maximal torus of $G(E)$. Since $p\left(T^{\prime}\right)=T$ and a surjective homomorphism from a torus to a torus always has a section (see Borel [1]), there is a section

$$
s: T \rightarrow T^{\prime} \subset G(E) .
$$

Let $E$ be a $T$-linearized vector bundle on an $X$ which is defined by a cone complex $(\Gamma, \mathscr{C})$. Let $C$ in $\mathscr{C}^{n}$ be a maximal cone and let $U$ be the corresponding $T$-stable affine open set in $X$, i.e. $U=\operatorname{Spec}(A)$ where $A=k[e(D)]$ is a polynomial ring with $D=C^{*} \cap \Xi . \quad A$ is a $E$-graded ring since there is a $T$-action on $A$. Namely

$$
A=\bigoplus_{\xi \in \varepsilon} A_{\xi}
$$

where $A_{\xi}$ consists of element $a$ of $A$ with

$$
a^{t}=e(\xi)(t) a
$$

$a^{t}$ is the translation of $a$ induced by the automorphism $t: U \rightarrow U$. We note that $A_{\xi}=k e(\xi)$ if $\xi \in D$ and $A_{\xi}=0$ if $\xi \notin D$.

Proposition 3.4. Let $D$ be a subsemi-group of $\Xi$ such that $A=$ $k[e(D)]$ is a polynomial ring. Let $M$ be an A-module. Then a $T$ linearization on the sheaf $\tilde{M}$ on $\operatorname{Spec}(A)$ coincides with a $\Xi$-graded $A$ - 
module structure on $M$.

Proof. Let $M$ be a $\Xi$-graded $A$-module, i.e.

$$
M=\bigoplus_{\xi \in \varepsilon} M_{\xi} .
$$

Then there is a $k$-isomorphism

$$
t^{*}: M \rightarrow M
$$

sending $m$ to $m^{t}=\sum_{\xi \in z} e(\xi)(t) m_{\xi}$ if $m=\sum_{\xi \in \varepsilon} m_{\xi}$ where $e(\xi)(t)$ is a value at $t$ of a function $e(\xi)$. Since $A$ is a $E$-graded module there exists a $k$-isomorphism

$$
t^{*}: A \rightarrow A
$$

sending $a$ to $a^{t}$. We denote $t^{*} A=A^{\prime}$. Let $A^{\prime}$ be an $A$-module through $t^{*}, A^{\prime} \otimes_{A} M$ is $A$-module. Furthermore $A^{\prime} \otimes_{A^{\prime}} M \simeq M$ by trivial isomorphism $A^{\prime} \simeq A$. So we have $t$-semi-linear $A$-isomorphism

$$
t^{*}: A^{\prime} \otimes_{A} M \rightarrow A^{\prime} \otimes_{A^{\prime}} M
$$

sending $a^{t} \otimes m$ to $a^{t} \otimes m^{t}$. Thus we have

$$
\phi_{t}: t^{*} \tilde{M} \rightarrow \tilde{M} \text {. }
$$

By construction, for every pair $\left(t, t^{\prime}\right),\left(t t^{\prime}\right)^{*}=t^{*} \cdot t^{*}$ means the relation

$$
\phi_{t t^{\prime}}=\phi_{t^{\prime}} \cdot t^{*} \phi_{t}
$$

So $\tilde{M}$ has a $T$-linearization. Conversely if $\tilde{M}$ has a $T$-linearization we can reverse the above order so $M$ is a $E$-graded module. Q.E.D.

$E \mid U$ is associated to a projective $A$-module $M$. Since $\tilde{M}$ has a $T$ linearization we see that $M$ is a $\Xi$-graded $A$-module. Let $D$ be a subsemi-group of $\Xi$ generated by a $Z$-base $\left\{\eta_{1}, \cdots, \eta_{n}\right\}$ of $E$, i.e. $D=Z_{0} \eta_{1}+$ $\cdots+Z_{0} \eta_{n}$, where $Z_{0}$ is the set of non-negative integers. Let $A=k[e(D)]$, i.e. $A=k\left[u_{1}, \cdots, u_{n}\right]$ is a polynomial ring of $n$-variables $u_{i}=e\left(\eta_{i}\right)(i=1$, $\cdots, n)$ with the $E$-gradation given by $\operatorname{deg}\left(u_{i}\right)=\eta_{i}$. For $\xi \in \Xi$ we denote by $A(\xi)$ the $E$-graded $A$-free module of rank one defined by

$$
A(\xi)_{\eta}=A_{\xi+\eta}
$$

THEOREM 3.5. Let $A=k[e(D)]$ be as above. If $M$ is a finitely generated $\Xi$-graded $A$-projective module of rank $r$, then there exist $\xi_{1}$, $\cdots, \xi_{r}$ in $\Xi$ such that 


$$
M \simeq A\left(-\xi_{1}\right) \oplus \cdots \oplus A\left(-\xi_{r}\right)
$$

as $E$-graded $A$-module, in particular $M$ is a free A-module.

Proof. Let $\left\{x_{1}, \cdots, x_{p}\right\}$ be a minimal set of generators of $M$ as an $A$-module. We may assume $x_{1}, \cdots, x_{p}$ are homogeneous with characters $\xi_{1}, \cdots, \xi_{p}$ respectively. Let

$$
F=\bigoplus_{i=1}^{p} A\left(-\xi_{i}\right)
$$

and let $e_{i}$ be the element of $A\left(-\xi_{i}\right)$ corresponding to 1 . We define a degree preserving $A$-homomorphism

$$
f: F \rightarrow M
$$

by $f\left(e_{i}\right)=x_{i}(i=1, \cdots, p), f$ is a surjective homomorphism of $E$ graded $A$-modules. Let $N$ be the kernel of $f$, hence we have an exact sequence

$$
0 \longrightarrow N \stackrel{i}{\longrightarrow} F \stackrel{f}{\longrightarrow} M \longrightarrow 0 \text {. }
$$

By renumbering if necessary, we may assume that

$$
\xi_{1}=\cdots=\xi_{q}, \quad \xi_{j} \notin \xi_{1}+D \quad \text { if } j>q .
$$

In fact put

$$
P=\left\{\xi_{i} \mid \xi_{i} \in \xi_{1}+D\right\} .
$$

If $\xi_{2} \in P$ and $\xi_{2} \neq \xi_{1}$ then there is non-zero $\eta$ in $D$ such that $\xi_{2}=\xi_{1}+\eta$. Put

$$
P^{\prime}=\left\{\xi_{i} \mid \xi_{i} \in \xi_{2}+D\right\} .
$$

Since $D$ is a semi-group with zero and with no subgroup we see that $P^{\prime} \subset P$ and $\xi_{1} \notin P^{\prime}$. Thus we replace $\xi_{1}$ by $\xi_{2}$. We do the same for this new $\xi_{1}$ and keep on doing the same. The process terminates since the original $P$ is finite.

We may assume $q<p$. In fact suppose $\xi_{1}=\cdots=\xi_{p}$ and let

$$
\sum_{i=1}^{p} a_{i} x_{i}=0
$$

be a homogeneous relation satisfied by $x_{i}=f\left(e_{i}\right)$ with $a_{i}$ homogeneous in $A$. Since $\operatorname{deg}\left(x_{i}\right)=\xi_{1}$ for all $i$, there exists an $\eta$ in $D$ such that 
$\operatorname{deg}\left(a_{i}\right)=\eta$ for all $i$. Hence we can write $a_{i}=k_{i} e(\eta)$ where $k_{i}$ are in $k$. Therefore our relation becomes

$$
e(\eta) \sum_{i=1}^{p} k_{i} x_{i}=0
$$

Since $M$ is $A$-flat we have $\sum_{i=1}^{p} k_{i} x_{i}=0$. If there is $i$ such that $k_{i} \neq 0$, then $x_{i}=-\sum_{j \neq i} k_{i}^{-1} k_{j} x_{j}$. This is a contradiction to the minimality of the set of generators $\left\{x_{1}, \cdots, x_{p}\right\}$. If $k_{i}=0$ for all $i$ then $a_{i}=0$ for all $i$. Hence $\left\{x_{i}, \cdots, x_{p}\right\}$ forms an $A$-free base of $M$, thus $M$ is free. Therefore we may assume that $q<p$.

We prove proposition 3.4 by induction on $\operatorname{rank}(F)=p$. If $\operatorname{rank}(F)$ $=1$ we are done. Thus suppose $\operatorname{rank}(F)>1$ and $q<\operatorname{rank}(F)$. Since $M$ is $A$-projective there is an $A$-module section $s: M \rightarrow F$ such that $f \cdot s$ $=1$. This means

$$
F=N \oplus s(M)
$$

as $A$-module. We want to show that $F=N \oplus s(M)$ as $E$-graded $A$ module by replacing $s$. Since $\operatorname{Hom}(\tilde{M}, \tilde{F})$ is $T$-linearized vector bundle $\operatorname{Hom}(M, F)$ is $E$-graded $A$-module. So we take $s_{0}$ as a section where $s_{0}$ is the degree 0 part of $s$. Since $\Xi$-graded homomorphism

$$
\operatorname{Hom}(M, F) \rightarrow \operatorname{Hom}(M, M)
$$

is surjective and sending $s$ to identity, so $s_{0}$ is not zero. So this $s_{0}$ satisfies the assertion.

We continue the proof of proposition 3.4. We may assume that $F=N \oplus s(M)$ as $E$-graded $A$-module. Since

$$
F_{\xi_{1}} \otimes_{k} A=A\left(-\xi_{1}\right) \oplus \cdots \oplus A\left(-\xi_{q}\right)
$$

by the choice of $\xi_{1}$ and

$$
(N \oplus s(M))_{\xi_{1}} \otimes_{k} A=\left(N_{\xi_{1}} \otimes_{k} A\right) \oplus\left(s(M)_{\xi_{1}} \otimes_{k} A\right)
$$

we have

$$
\begin{aligned}
A\left(-\xi_{q+1}\right) \oplus \cdots \oplus A\left(-\xi_{p}\right) & =F / F_{\xi_{1}} \otimes_{k} A \\
& =(N \oplus s(M)) /\left\{\left(N_{\xi_{1}} \otimes_{k} A\right) \oplus\left(s(M)_{\xi_{1}} \otimes_{k} A\right)\right\} \\
& =\left(N / N_{\xi_{1}} \otimes_{k} A\right) \oplus\left(s(M) / s(M)_{\xi_{1}} \otimes_{k} A\right) .
\end{aligned}
$$

Since $s(M) / s(M)_{\xi_{1}} \otimes_{k} A$ is a direct summand of $A$-free module 


$$
A\left(-\xi_{q+1}\right) \oplus \cdots \oplus A\left(-\xi_{p}\right)
$$

it is $A$-projective. Thus, by induction assumption, we have

$$
s(M) / s(M)_{\xi_{1}} \otimes_{k} A=A\left(-\xi_{1}^{\prime}\right) \oplus \cdots \oplus A\left(-\xi_{m}^{\prime}\right)
$$

is a free $A$-module for some $\xi_{i}^{\prime}$. Since $s(M)_{\xi_{1}} \otimes_{k} A$ is $A$ free we have

$$
M \simeq s(M)=A\left(-\xi_{1}^{\prime}\right) \oplus \cdots \oplus A\left(-\xi_{m}^{\prime}\right) \oplus\left(s(M)_{\xi_{1}} \otimes_{k} A\right)
$$

is a free module of rank $r$.

Q.E.D.

Let $\left(\tilde{M}, \phi_{t}\right)$ be a $T$-linearized vector bundle on $U=\operatorname{Spec}(A)$ with $A=k[e(D)]$ for some subsemi-group $D$ of $E$. We say that $m \in M=$ $\Gamma(U, \tilde{M})$ is semi-invariant if there exists a character $\xi \in \Xi$ such that

$$
\phi_{t}(m)=e(\xi)(t) m
$$

is satisfied for every $t$, i.e. $m$ is homogeneous element of degree $\xi$ of $M$.

CoRollary 3.6. Let $\left(\tilde{M}, \phi_{t}\right)$ be as above. Then $\tilde{M}$ has a semi-invariant base.

Proof. By the theorem there exist characters $\xi_{1}, \cdots, \xi_{r}$ so that

$$
M=A\left(-\xi_{1}\right) \oplus \cdots \oplus A\left(-\xi_{r}\right) .
$$

Let $e_{i}$ be the element of $A\left(-\xi_{i}\right)$ corresponding to 1 . We denote by the same letter the element of $M$ corresponding to $e_{i}$. Then we can easily see that $e_{i}$ is semi-invariant. Furthermore $\left\{e_{i}\right\}$ is a base of $\tilde{M}$ as $\tilde{A}$ module.

Q.E.D.

\section{\$ 4. Construction of an equivariant vector bundle}

Let $(X, T)$ be a smooth complete almost homogeneous variety defined by the cone complex $(\Gamma, \mathscr{C})$. Let $E$ be a $T$-linearized vector bundle of rank $r$ on $X$. We have shown that $E \mid U$ has a semi-invariant base where $U$ is a $T$-stable affine open subset of $X$. Let $U$ and $U^{\prime}$ be $T$-stable affine open subsets corresponding to $C$ and $C^{\prime}$ in $\mathscr{C}^{n}$ respectively where $\mathscr{C}^{n}$ is the set of $n$-dimensional (maximal) cones of $\mathscr{C}$. Let $\left(u_{i}\right)$ and $\left(u_{j}^{\prime}\right)$ be semi-invariant bases on $E(U)$ and $E\left(U^{\prime}\right)$ respectively and let $\left(\xi_{i}\right)$ and $\left(\xi_{j}^{\prime}\right)$ be corresponding characters respectively. There is a natural pairing $\langle\rangle:, \Xi \times \Gamma \rightarrow Z$. It can naturally be extended to $\boldsymbol{\Xi}_{\boldsymbol{Q}} \times \Gamma_{\boldsymbol{Q}} \rightarrow \boldsymbol{Q}$. We denote $\phi(\xi)=\langle\xi, \phi\rangle$ for $\xi \in \Xi_{Q}$ and $\phi \in \Gamma_{Q}$. 
Proposition 4.1. In the above situation there is a permutation $\sigma$ such that $\phi\left(\xi_{i}\right)=\phi\left(\xi_{o(i)}^{\prime}\right)$ for every $\phi$ in $C \cap C^{\prime}$.

Proof. We note $T \subset X$. Since $U \supset T$ and $U^{\prime} \supset T$ we have

$$
E|U| T \simeq E\left|U^{\prime}\right| T
$$

We denote

$$
\begin{aligned}
M & =E(U)=A u_{1} \oplus \cdots \oplus A u_{r} \simeq A\left(-\xi_{1}\right) \oplus \cdots \oplus A\left(-\xi_{r}\right) \\
M^{\prime} & =E\left(U^{\prime}\right)=A^{\prime} u_{1}^{\prime} \oplus \cdots \oplus A^{\prime} u_{r}^{\prime} \simeq A^{\prime}\left(-\xi_{1}^{\prime}\right) \oplus \cdots \oplus A^{\prime}\left(-\xi_{r}^{\prime}\right)
\end{aligned}
$$

where $A=k\left[e\left(C^{*} \cap \Xi\right)\right]$ and $A^{\prime}=k\left[e\left(C^{*} \cap E\right)\right]$ are affine rings corresponding to $U$ and $U^{\prime}$ respectively. The above isomorphism restricted to $T$ induces the isomorphism

$$
k[T] \otimes_{A} M \simeq k[T] \otimes_{A^{\prime}} M^{\prime}
$$

as $E$-graded $k[T]$-modules. Since

$$
\begin{aligned}
& k[T] \otimes_{A} M \simeq k[T] \otimes_{k}\left(\bigoplus_{i=1}^{r} k e\left(-\xi_{i}\right) u_{i}\right) \\
& k[T] \otimes_{A^{\prime}} M^{\prime} \simeq k[T] \otimes_{k}\left(\bigoplus_{j=1}^{r} k e\left(-\xi_{j}^{\prime}\right) u_{j}^{\prime}\right)
\end{aligned}
$$

we have $k$-isomorphism

$$
\bigoplus_{i=1}^{r} k e\left(-\xi_{i}\right) u_{i} \rightarrow \bigoplus_{j=1}^{r} k e\left(-\xi_{j}^{\prime}\right) u_{j}^{\prime}
$$

i.e. there is a matrix $P=P\left(C, C^{\prime}\right)$ in $G L_{r}(k)$ such that

$$
\left(\begin{array}{c}
e\left(-\xi_{1}\right) u_{1} \\
\vdots \\
e\left(-\xi_{r}\right) u_{r}
\end{array}\right)=P\left(\begin{array}{c}
e\left(-\xi_{1}^{\prime}\right) u_{1}^{\prime} \\
\vdots \\
e\left(-\xi_{r}^{\prime}\right) u_{r}^{\prime}
\end{array}\right) \text {. }
$$

Then

$$
\begin{aligned}
& \left(\begin{array}{c}
u_{1} \\
\vdots \\
u_{r}
\end{array}\right)=\left(\begin{array}{ccc}
e\left(-\xi_{1}\right) & & \\
& \ddots & 0 \\
0 & & e\left(-\xi_{r}\right)
\end{array}\right)^{-1} P\left(\begin{array}{ccc}
e\left(-\xi_{1}^{\prime}\right) & & \\
& \ddots & 0 \\
0 & & e\left(-\xi_{r}^{\prime}\right)
\end{array}\right)\left(\begin{array}{c}
u_{1}^{\prime} \\
\vdots \\
u_{r}^{\prime}
\end{array}\right) \\
& =\left(p_{i j} e\left(\xi_{i}-\xi_{j}^{\prime}\right)\right)\left(\begin{array}{c}
u_{1}^{\prime} \\
\vdots \\
u_{r}^{\prime}
\end{array}\right)
\end{aligned}
$$


where $p_{i j}$ is the $(i, j)$-entry of $P$. Since, on $U \cap U^{\prime}, p_{i j} e\left(\xi_{i}-\xi_{j}^{\prime}\right)$ are regular functions

$$
\phi\left(\xi_{i}\right) \geqq \phi\left(\xi_{j}^{\prime}\right)
$$

for every $\phi$ in $C \cap C^{\prime}$ if $p_{i j} \neq 0$. Since

$$
\operatorname{det} P=\sum_{\sigma \in \mathbb{E}_{\boldsymbol{r}}} \operatorname{sgn}(\sigma) p_{1_{\sigma(1)}} \cdots p_{r_{\boldsymbol{\sigma}}(r)} \neq 0
$$

there exists a permutation $\sigma$ such that $p_{i_{\sigma(i)}} \neq 0$ for every $i$. Thus

$$
\phi\left(\xi_{i}\right) \geqq \phi\left(\xi_{\sigma(i)}^{\prime}\right)
$$

for every $i$ and every $\phi$ in $C \cap C^{\prime}$. Furthermore since

$$
\operatorname{det}\left(p_{i j} e\left(\xi_{i}-\xi_{j}^{\prime}\right)\right)=\operatorname{det}(P) e\left(\xi_{i}+\cdots+\xi_{r}-\xi_{1}^{\prime}-\cdots-\xi_{r}^{\prime}\right)
$$

is a unit on $U \cap U^{\prime}$, we have

$$
\phi\left(\xi_{1}+\cdots+\xi_{r}-\xi_{1}^{\prime}-\cdots-\xi_{r}^{\prime}\right)=0
$$

for every $\phi$ in $C \cap C^{\prime}$. Compairing this equality with the above inequality, we have

$$
\phi\left(\xi_{i}\right)=\phi\left(\xi_{\sigma(i)}^{\prime}\right)
$$

for every $i$ and every $\phi$ in $C \cap C^{\prime}$.

By virtue of Proposition 4.1, a T-linearized vector bundle of rank $r$ on $X$ gives rise to the following data:

(i ) $m: S k^{1}(\mathscr{C})=\mathscr{C}^{1}=\left\{\phi_{1}, \cdots, \phi_{d}\right\} \rightarrow Z^{\oplus r}$

sending $\phi$ to $m(\phi)=\left(m(\phi)_{1}, \cdots, m(\phi)_{r}\right)$ where $S k^{1}(\mathscr{C})$ is a set of 1-dimensional cones of $\mathscr{C}$, and for every $C$ in $\mathscr{C}^{n}$

$$
m_{C}: C \cap S k^{1}(\mathscr{C}) \rightarrow Z^{\oplus r}
$$

so that there is a permutation $\tau$ such that

$$
\begin{aligned}
m_{C}(\phi) & =\left(m_{C}(\phi)_{1}, \cdots, m_{C}(\phi)_{r}\right) \\
& =\left(m(\phi)_{\tau(1)}, \cdots, m(\phi)_{\tau(r)}\right)
\end{aligned}
$$

for every $\phi$ in $C \cap S k^{1}(\mathscr{C})$.

Suppose the data (i) are given, then for $C$ in $\mathscr{C}^{n}$ we have characters $\xi(C)_{i}$ by solving equations

$$
\phi(\xi)=m_{C}(\phi)_{i}
$$


for every $\phi$ in $C \cap S k^{1}(\mathscr{C})$. For maximal cones $C$ and $C^{\prime}$ there exists a permutation $\tau$ such that

$$
\phi\left(\xi(C)_{i}\right)=\phi\left(\xi\left(C^{\prime}\right)_{\tau(i)}\right)
$$

for every $i$ and every $\phi$ in $C \cap C^{\prime}$ by the condition on $m_{C}$ and $m_{C^{\prime}}$ in (i). Conversely if we have these $\xi(C)_{i}$ then the data (i) is obtained. So (i) is equivalent to

(i') $\xi: \mathscr{C}^{n} \rightarrow \Xi^{\oplus r}$

sending $C$ to $\xi(C)=\left(\xi(C)_{1}, \cdots, \xi(C)_{r}\right)$ such that for every pair of cones $C, C^{\prime}$ in $\mathscr{C}^{n}$ there exists a permutation $\tau$ so that $\phi\left(\xi(C)_{i}\right)=\phi\left(\xi\left(C^{\prime}\right)_{\tau(i)}\right) \quad$ for every $i$ and every $\phi$ in $C \cap C^{\prime}$.

(ii) $P: \mathscr{C}^{n} \times \mathscr{C}^{n} \rightarrow G L_{r}(k)$ sending $\left(C, C^{\prime}\right)$ to $P\left(C, C^{\prime}\right)=\left(P\left(C, C^{\prime}\right)_{i j}\right)$ such that $P\left(C, C^{\prime}\right)_{i j} \neq 0$ only if $m_{C}(\phi)_{i} \geqq m_{C^{\prime}}(\phi)_{j}$ for every $\phi$ in $\left(C \cap C^{\prime}\right) \cap S k^{1}(\mathscr{C})$ and such that

$$
P\left(C, C^{\prime}\right) P\left(C^{\prime}, C^{\prime \prime}\right)=P\left(C, C^{\prime \prime}\right)
$$

for every $C, C^{\prime}, C^{\prime \prime}$ in $\mathscr{C}^{n}$.

(iii) For two pairs $(m, P)$ and $\left(m^{\prime}, P^{\prime}\right)$, we say that they are equivalent if there exists a permutation $\sigma=\sigma(C)$ in $\widetilde{S}_{r}$ such that

$$
\left(m_{C}(\phi)_{1}, \cdots, m_{C}(\phi)_{r}\right)=\left(m_{C}^{\prime}(\phi)_{\sigma(1)}, \cdots, m_{C}^{\prime}(\phi)_{\sigma(r)}\right)
$$

for every $C$ in $\mathscr{C}^{n}$ and $\phi$ in $C \cap S k^{1}(\mathscr{C})$ and if there exists

$$
\rho: \mathscr{C}^{n} \rightarrow G L_{r}(k)
$$

such that

$$
P^{\prime}\left(C, C^{\prime}\right)=\rho(C)^{-1} P\left(C, C^{\prime}\right) \rho\left(C^{\prime}\right)
$$

for every $C$ and $C^{\prime}$ in $\mathscr{C}^{n}$.

THEOREM 4.2. Let $(X, T)$ be a smooth complete almost homogeneous variety defined by a cone complex $(\Gamma, \mathscr{C})$. The set of T-linearized vector bundles of rank $r$ up to T-isomorphism corresponds bijectively to the set of data (i') and (ii) up to equivalence (iii).

Proof. Let $\left(E, \phi_{t}\right)$ be a $T$-linearized vector bundle of rank $r$ on $X$. Let $U$ be an affine open subset of $X$ corresponding to $C$ in $\mathscr{C}^{n}$. Let $\left(u_{i}\right)$ be semi-invariant base of $E \mid U$ on $U$ and let $\left(\xi_{i}\right)$ be characters corresponding to $\left(u_{i}\right)$. We define $m_{C}$ by 


$$
m_{C}(\phi)_{i}=\phi\left(\xi_{i}\right)=\left\langle\xi_{i}, \phi\right\rangle \quad i=1, \cdots, r
$$

for every $\phi$ in $C \cap S k^{1}(\mathscr{C})$. Then the condition of (i) are satisfied. $P$ is obtained in Proposition 4.1. Let $\left(F, \psi_{t}\right)$ be a $T$-linearized vector bundle of rank $r$ on $X$. Let $\left(v_{j}\right)$ be semi-invariant base of $F \mid U$ on $U$ and let $\left(\eta_{j}\right)$ be characters corresponding to $\left(v_{j}\right)$. Suppose $E$ and $F$ are $T$-isomorphic. We denote

$$
\begin{aligned}
M & =E(U)=A u_{1} \oplus \cdots \oplus A u_{r} \\
N & =F(U)=A v_{1} \oplus \cdots \oplus A v_{r}
\end{aligned}
$$

where $A=A_{C}$ is a polynomial ring corresponding to the cone $C$. Since $E|U \simeq F| U$ we have $E$-graded $A$-isomorphism

$$
M=A \otimes\left(\bigoplus_{i=1}^{r} k e\left(-\xi_{i}\right) u_{i}\right) \rightarrow N=A \otimes\left(\bigoplus_{j=1}^{r} k e\left(-\eta_{j}\right) v_{j}\right) .
$$

So we have $k$-isomorphism

$$
\bigoplus_{i=1}^{r} k e\left(-\xi_{i}\right) u_{i} \rightarrow \bigoplus_{j=1}^{r} k e\left(-\eta_{j}\right) v_{j}
$$

i.e. there exists a matrix $\rho=\rho(C)$ in $G L_{r}(k)$ such that

$$
\left(\begin{array}{c}
e\left(-\xi_{1}\right) u_{1} \\
\vdots \\
e\left(-\xi_{r}\right) u_{r}
\end{array}\right)=\rho\left(\begin{array}{c}
e\left(-\eta_{1}\right) v_{1} \\
\vdots \\
e\left(-\eta_{r}\right) v_{r}
\end{array}\right) \text {. }
$$

Then

$$
\left(\begin{array}{c}
u_{1} \\
\vdots \\
u_{r}
\end{array}\right)=\left(\rho_{i j} e\left(\xi_{i}-\eta_{\jmath}\right)\right)\left(\begin{array}{c}
v_{1} \\
\vdots \\
v_{r}
\end{array}\right)
$$

where $\rho_{i j}$ is the $(i, j)$-entry of $\rho$. Since, on $U, \rho_{i j} e\left(\xi_{i}-\eta_{j}\right)$ are regular functions we have

$$
\phi\left(\xi_{i}\right) \geqq \phi\left(\eta_{j}\right)
$$

for every $\phi$ in $C$ if $\rho_{i j} \neq 0$. Since

$$
\operatorname{det}(\rho)=\sum_{\sigma \in \mathscr{E}_{n}} \operatorname{sgn}(\sigma) \times \rho_{i_{\sigma(i)}} \cdots \rho_{r \sigma(r)} \neq 0
$$

there exists a permutation $\sigma=\sigma(C)$ such that $\rho_{i_{\sigma}(i)} \neq 0$ for every $i$. Thus

$$
\phi\left(\xi_{i}\right) \geqq \phi\left(\eta_{\sigma(i)}\right)
$$


for every $i$ and every $\phi$ in $C$. Furthermore since

$$
\operatorname{det}\left(\rho_{i j} e\left(\xi_{i}-\eta_{j}\right)\right)=\operatorname{det}(\rho) e\left(\xi_{1}+\cdots+\xi_{r}-\eta_{1}-\cdots-\eta_{r}\right)
$$

is a unit on $U$, we have

$$
\phi\left(\xi_{1}\right)+\cdots+\phi\left(\xi_{r}\right)=\phi\left(\eta_{\sigma(1)}\right)+\cdots+\phi\left(\eta_{\sigma(r)}\right)
$$

for every $\phi$ in $C$. So

$$
\phi\left(\xi_{i}\right)=\phi\left(\eta_{\sigma(i)}\right)
$$

for every $\phi$ in $C$. So this $\sigma$ satisfies the condition of $\sigma(C)$ of (iii). Let $U^{\prime}$ be an affine open subset of $X$ corresponding to $C^{\prime}$ in $\mathscr{C}^{n}$. Consider the diagram on $U \cap U^{\prime}$

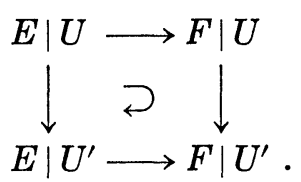

Let $P=P\left(C, C^{\prime}\right)$ be defined in Proposition 4.1 for $E$ and let $Q=Q\left(C, C^{\prime}\right)$ for $F$. Then by virtue of above commutative diagram we have

$$
\rho(C) P\left(C, C^{\prime}\right)=Q\left(C, C^{\prime}\right) \rho\left(C^{\prime}\right)
$$

where $\rho$ is defined above. This means the second part of (iii).

Conversely if we have the data (i') and (ii), then the $T$-linearized vector bundle $E$ of rank $r$ can be constructed as follows. For $C$ in $\mathscr{C}^{n}$, we write by $U_{C}=A^{n}=\operatorname{Spec}\left(A_{C}\right)$ a $T$-stable affine open subset of $X$ corresponding to $C$. Suppose $m$ and $P$ are given. Let

$$
E\left(U_{C}\right)=A_{C}\left(-\xi(C)_{1}\right) \oplus \cdots \oplus A_{C}\left(-\xi(C)_{r}\right)
$$

for each $C$ in $\mathscr{C}^{n}$. The $\Xi$-graded $A_{C}$-module structure on $E\left(U_{C}\right)$ gives rise to a $T$-linearized vector bundle $E \mid U_{C}=\overparen{E\left(U_{C}\right)}$ on $U_{C}$. Let

$$
f_{C, C^{\prime}}: A_{C \cap C^{\prime}} \otimes_{A_{C}} E\left(U_{C}\right) \rightarrow A_{C^{\prime} \cap C} \otimes_{A_{C^{\prime}}} E\left(U_{C^{\prime}}\right)
$$

be $\boldsymbol{E}$-graded $A_{C \cap C^{\prime}}$-module isomorphism defined by

$$
\operatorname{diag}\left(e\left(\xi\left(C^{\prime}\right)_{1}\right), \cdots, e\left(\xi\left(C^{\prime}\right)_{r}\right)\right) P\left(C, C^{\prime}\right)^{-1} \operatorname{diag}\left(e\left(-\xi(C)_{1}\right), \cdots, e\left(-\xi(C)_{r}\right)\right) .
$$

This $f_{C, C^{\prime}}$ gives rise to an isomorphism

$$
f_{C, C^{\prime}}: E\left|U_{C}\right| U_{C} \cap U_{C^{\prime}} \rightarrow E\left|U_{C^{\prime}}\right| U_{C^{\prime}} \cap U_{C}
$$


compatible with the action of $T$. By the condition of (ii) we can patch $E \mid U_{C}$ and $E \mid U_{C^{\prime}}$ along $U_{C} \cap U_{C^{\prime}}$. So we obtain a $T$-linearized vector bundle $E=E(m, P)$ on $X$. The data $(m, P)$ is equivalent to the data $\left(m^{\prime}, P^{\prime}\right)$ then by the construction $E(m, P)$ and $E\left(m^{\prime}, P^{\prime}\right)$ are $T$-isomorphic $T$-linearized vector bundles.

Q.E.D.

Remark 4.3. Two $T$-linearized vector bundles $E(m, P)$ of rank $r$ and $E\left(m^{\prime}, P^{\prime}\right)$ of rank $r^{\prime}$ are given. Then the $T$-linearized vector bundle $E(m, P) \otimes E\left(m^{\prime}, P^{\prime}\right)$ is $E\left(m \otimes m^{\prime}, P \otimes P^{\prime}\right)$ where

$$
\left(m \otimes m^{\prime}\right)(\phi)=\left(\cdots, m(\phi)_{i}+m^{\prime}(\phi)_{j}, \cdots\right)
$$

and $P \otimes P^{\prime}$ means the Kronecker product. The $T$-linearized vector bundle $\operatorname{det} E(m, P)$ is $E(\operatorname{det} m, \operatorname{det} P)$ where

$$
(\operatorname{det} m)(\phi)=\sum_{i=1}^{r} m(\phi)_{i}
$$

and

$$
(\operatorname{det} P)\left(C, C^{\prime}\right)=\operatorname{det}\left(P\left(C, C^{\prime}\right)\right) \text {. }
$$

Remark 4.4. The case of rank $=1$. For $\phi_{i} \in S k^{1}(\mathscr{C})$ we denote by $m_{i}$ the value $m\left(\phi_{i}\right)$. Let $D_{i}$ be the divisor corresponding to $\phi_{i}$ i.e. $D_{i}=\operatorname{div}\left(e\left(\xi_{i}\right)\right)$ on $T$-stable affine open $U_{k}$ where $\xi_{i}$ is a character so that $\phi_{j}\left(\xi_{i}\right)=\delta_{j i}$ for $\phi_{j} \in S k^{1}(\mathscr{C}) \cap C_{k}$. Then the data $m=\left(m_{i}\right)$ corresponds to the line bundle $O_{X}\left(-\sum m_{i} D_{i}\right)$.

\section{§5. Examples on $\boldsymbol{P}^{2}$}

In this section, we consider $X=P^{2}=\operatorname{Proj}\left(k\left[X_{0}, X_{1}, X_{2}\right]\right)$ with the standard action of $T=G_{m} \times G_{m}$. Let $E(a, b, c)$ be a vector bundle defined by the exact sequence

$$
\begin{aligned}
0 \rightarrow O_{X} & \rightarrow O_{X}(a) \oplus O_{X}(b) \oplus O_{X}(c) \rightarrow E(a, b, c)^{*} \rightarrow 0 \\
1 & \rightarrow\left(X_{0}^{a}, X_{1}^{b}, X_{2}^{c}\right)
\end{aligned}
$$

where $a, b, c$ are positive integers. It is easy to see that $E(a, b, c)^{*}$ is an equivariant vector bundle for positive integers $a, b, c$.

THEOREM 5.1. Let $T$ be a 2-dimensional torus. $T$ acts naturally on $\boldsymbol{P}^{2}$ and it becomes an almost homogeneous variety. An indecomposable equivariant vector bundle of rank 2 on $\boldsymbol{P}^{2}$ is isomorphic to $E(a, b, c) \otimes$ $O_{p^{2}}(n)$ for some integer $n$ and some positive integers $a, b, c$. 
We now compute the data $(m, P)$ for $E(a, b, c)$. Put $S=k\left[X_{0}, X_{1}, X_{2}\right]$ and let $M$ be the kernel of

$$
\begin{aligned}
S e_{0} \oplus S e_{1} \oplus S e_{2} & \rightarrow S \\
e_{0} & \mapsto X_{0}^{a} \\
e_{1} & \mapsto X_{1}^{b} \\
e_{2} & \mapsto X_{2}^{c} .
\end{aligned}
$$

The generators of $M$ are

$$
X_{1}^{b} e_{0}-X_{0}^{a} e_{1}, \quad X_{1}^{b} e_{2}-X_{2}^{c} e_{1}, \quad X_{0}^{a} e_{2}-X_{2}^{c} e_{0} .
$$

Put $x=X_{0} / X_{2}, y=X_{1} / X_{2}$ and let $U_{1}, U_{2}, U_{3}$ be affine spaces defined by $X_{2} \neq 0, X_{0} \neq 0, X_{1} \neq 0$ respectively, i.e.,

$$
U_{1}=\operatorname{Spec}(k[x, y]), \quad U_{2}=\operatorname{Spec}\left(k\left[\frac{1}{x}, \frac{y}{x}\right]\right), \quad U_{3}=\operatorname{Spec}\left(k\left[\frac{1}{y}, \frac{x}{y}\right]\right) .
$$

Put

$$
\begin{array}{ll}
u_{1}=\frac{e_{1}}{X_{2}^{b}}-\left(\frac{X_{1}}{X_{2}}\right)^{b} \frac{e_{2}}{X_{2}^{c}}, & v_{1}=\frac{e_{0}}{X_{2}^{a}}-\left(\frac{X_{0}}{X_{2}}\right)^{a} \frac{e_{2}}{X_{2}^{c}}, \\
u_{2}=\frac{e_{1}}{X_{0}^{b}}-\left(\frac{X_{1}}{X_{0}}\right)^{b} \frac{e_{0}}{X_{0}^{a}}, & v_{2}=-\frac{e_{2}}{X_{0}^{c}}+\left(\frac{X_{2}}{X_{0}}\right)^{c} \frac{e_{0}}{X_{0}^{a}}, \\
u_{3}=-\frac{e_{0}}{X_{1}^{a}}+\left(\frac{X_{0}}{X_{1}}\right)^{a} \frac{e_{1}}{X_{1}^{b}}, & v_{3}=-\frac{e_{2}}{X_{1}^{c}}+\left(\frac{X_{2}}{X_{1}}\right)^{c} \frac{e_{1}}{X_{1}^{b}} .
\end{array}
$$

Let $t=(\lambda, \mu)$ in $T$ be acts by $t X_{0}=\lambda X_{0}, t X_{1}=\mu X_{1}, t X_{2}=X_{2}$. Then

$$
t e_{0}=\lambda^{a} e_{0}, \quad t e_{1}=\mu^{b} e_{1}, \quad t e_{3}=e_{3},
$$

and

$$
t x=\lambda x, \quad t y=\mu y .
$$

In this case we take semi-invariant basis $\left(u_{i}, v_{i}\right)$ on $U_{i}$ and we have

$$
\begin{aligned}
\left(\begin{array}{cc}
x^{b} y^{-b} & 0 \\
0 & x^{c}
\end{array}\right)\left(\begin{array}{l}
u_{2} \\
v_{2}
\end{array}\right) & =\left(\begin{array}{cc}
1 & -1 \\
0 & 1
\end{array}\right)\left(\begin{array}{cc}
y^{-b} & 0 \\
0 & x^{-a}
\end{array}\right)\left(\begin{array}{l}
u_{1} \\
v_{1}
\end{array}\right), \\
\left(\begin{array}{cc}
x^{-a} y^{a} & 0 \\
0 & y^{c}
\end{array}\right)\left(\begin{array}{l}
u_{3} \\
v_{3}
\end{array}\right) & =\left(\begin{array}{cc}
1 & 0 \\
1 & 1
\end{array}\right)\left(\begin{array}{cc}
x^{b} y^{-b} & 0 \\
0 & x^{c}
\end{array}\right)\left(\begin{array}{l}
u_{2} \\
v_{2}
\end{array}\right), \\
\left(\begin{array}{cc}
y^{-b} & 0 \\
0 & x^{-a}
\end{array}\right)\left(\begin{array}{l}
u_{1} \\
v_{1}
\end{array}\right) & =\left(\begin{array}{cc}
0 & 1 \\
-1 & 1
\end{array}\right)\left(\begin{array}{cc}
x^{-a} y^{a} & 0 \\
0 & y^{c}
\end{array}\right)\left(\begin{array}{l}
u_{3} \\
v_{3}
\end{array}\right) .
\end{aligned}
$$

Let $\phi_{1}, \phi_{2} \in \Gamma$ be such that 


$$
\phi_{1}(\lambda)=\phi_{2}(\mu)=1, \quad \phi_{1}(\mu)=\phi_{2}(\lambda)=0
$$

where $\lambda, \mu$ are characters of $T$. Then the decomposition of $\Gamma_{Q}$ by $\left(\phi_{1}, \phi_{2}, \phi_{3}=-\phi_{1}-\phi_{2}\right)$ defines a cone complex corresponding to $\boldsymbol{P}^{2}$ (see [4]) i.e. put

$$
\begin{aligned}
& C_{1}=\left\{p \phi_{1}+q \phi_{2} \mid p, q \in \boldsymbol{Q}_{0}\right\} \\
& C_{2}=\left\{p \phi_{2}+q \phi_{3} \mid p, q \in \boldsymbol{Q}_{0}\right\} \\
& C_{3}=\left\{p \phi_{3}+q \phi_{1} \mid p, q \in \boldsymbol{Q}_{0}\right\}
\end{aligned}
$$

then $\Gamma_{Q}=C_{1} \cup C_{2} \cup C_{3}$, and $C_{i}$ corresponds to an affine space $U_{i}$. Let $\xi_{i}, \eta_{i}$ be the characters corresponding to $u_{i}, v_{i}$ respectively i.e.

$$
\begin{array}{ll}
\xi_{1}(\lambda, \mu)=\mu^{b}, & \eta_{1}(\lambda, \mu)=\lambda^{a}, \\
\xi_{2}(\lambda, \mu)=\lambda^{-b} \mu^{b}, & \eta_{2}(\lambda, \mu)=\lambda^{-c}, \\
\xi_{3}(\lambda, \mu)=\lambda^{a} \mu^{-a}, & \eta_{3}(\lambda, \mu)=\mu^{-c} .
\end{array}
$$

Then

$$
\begin{array}{ll}
\phi_{1}\left(\xi_{1}\right)=\phi_{1}\left(\eta_{3}\right)=0, & \phi_{1}\left(\eta_{1}\right)=\phi_{1}\left(\xi_{3}\right)=a, \\
\phi_{2}\left(\xi_{2}\right)=\phi_{2}\left(\xi_{1}\right)=b, & \phi_{2}\left(\eta_{2}\right)=\phi_{2}\left(\eta_{1}\right)=0, \\
\phi_{3}\left(\xi_{3}\right)=\phi_{3}\left(\xi_{2}\right)=0, & \phi_{3}\left(\eta_{3}\right)=\phi_{3}\left(\eta_{2}\right)=c .
\end{array}
$$

These integers mean the data $m$ in $\S 4$, i.e.

$$
m\left(\phi_{1}\right)=(0, a), \quad m\left(\phi_{2}\right)=(b, 0), \quad m\left(\phi_{3}\right)=(0, c) .
$$

To prove Theorem 5.1 we have only to show that the data in $\S 4$ define a vector bundle

$$
E(a, b, c) \otimes O_{P 2}(n)
$$

for some integer $n$ and some positive integers $a, b, c$. Let $D_{i}$ be the divisor corresponding to $\phi_{i}$. By Remarks 4.3 and 4.4, the data $\bar{m}$ for

$$
E \otimes O_{P^{2}}\left(\sum_{i=1}^{3} m_{i} D_{i}\right)
$$

are

$$
\begin{array}{ll}
\bar{m}\left(\phi_{1}\right)=\left(m\left(\phi_{1}\right)_{1}-m_{1},\right. & \left.m\left(\phi_{1}\right)_{2}-m_{1}\right) \\
\bar{m}\left(\phi_{2}\right)=\left(m\left(\phi_{2}\right)_{1}-m_{2},\right. & \left.m\left(\phi_{2}\right)_{2}-m_{2}\right) \\
\bar{m}\left(\phi_{3}\right)=\left(m\left(\phi_{3}\right)_{1}-m_{3},\right. & \left.m\left(\phi_{3}\right)_{2}-m_{3}\right)
\end{array}
$$

where $m\left(\phi_{1}\right)=\left(m\left(\phi_{i}\right)_{1}, m\left(\phi_{i}\right)_{2}\right)$ are the data $m$ for $T$-linearized vector bundle 
E. Thus by tensoring

$$
O_{P^{2}}\left(m_{1}+m_{2}+m_{3}\right)=O_{P^{2}}\left(\sum_{i=1}^{3} m_{i} D_{i}\right)
$$

with the $T$-linearized vector bundle $E$, if necessary, we may assume that the data $m$ for $E$ are

$$
m\left(\phi_{i}\right)=\left(\alpha_{i}, 0\right) \quad \text { or } \quad m\left(\phi_{i}\right)=\left(0, \alpha_{i}\right)
$$

for non-negative integers $\alpha_{i}$. Furthermore by changing the base if necessary, we may assume that

$$
m_{C_{1}}\left(\phi_{2}\right)=m_{C_{2}}\left(\phi_{2}\right)=\left(\alpha_{2}, 0\right)
$$

and one of
a) $m_{C_{2}}\left(\phi_{3}\right)=m_{C_{3}}\left(\phi_{3}\right)=\left(\alpha_{3}, 0\right)$
b) $m_{C_{2}}\left(\phi_{3}\right)=m_{C_{3}}\left(\phi_{3}\right)=\left(0, \alpha_{3}\right)$

and one of

1) $m_{C_{3}}\left(\phi_{1}\right)=m_{C_{1}}\left(\phi_{1}\right)=\left(\alpha_{1}, 0\right)$

2) $m_{C_{3}}\left(\phi_{1}\right)=m_{C_{1}}\left(\phi_{1}\right)=\left(0, \alpha_{1}\right)$

3) $m_{C_{3}}\left(\phi_{1}\right)=\left(\alpha_{1}, 0\right), m_{C_{1}}\left(\phi_{1}\right)=\left(0, \alpha_{1}\right)$

4) $m_{C_{3}}\left(\phi_{1}\right)=\left(0, \alpha_{1}\right), m_{C_{1}}\left(\phi_{1}\right)=\left(\alpha_{1}, 0\right)$.

We note that if the data $P$ are of the form

$$
\left(\begin{array}{ll}
* & 0 \\
0 & *
\end{array}\right)
$$

i.e. $P\left(C_{1}, C_{2}\right), P\left(C_{2}, C_{3}\right), P\left(C_{3}, C_{1}\right)$ are of the above form, then the vector bundle $E(m, P)$ is decomposable.

If one of $\alpha_{i}$ is zero, we may assume $\alpha_{1}=0$, then
a) $\quad P\left(C_{1}, C_{2}\right)=\left(\begin{array}{ll}a & b \\ 0 & d\end{array}\right), \quad P\left(C_{2}, C_{3}\right)=\left(\begin{array}{ll}a^{\prime} & b^{\prime} \\ 0 & d^{\prime}\end{array}\right), \quad P\left(C_{3}, C_{1}\right)=\left(\begin{array}{ll}a^{\prime \prime} & b^{\prime \prime} \\ c^{\prime \prime} & d^{\prime \prime}\end{array}\right)$

or

b) $P\left(C_{1}, C_{2}\right)=\left(\begin{array}{ll}a & b \\ 0 & d\end{array}\right), \quad P\left(C_{2}, C_{3}\right)=\left(\begin{array}{ll}a^{\prime} & 0 \\ c^{\prime} & d^{\prime}\end{array}\right), \quad P\left(C_{3}, C_{1}\right)=\left(\begin{array}{ll}a^{\prime \prime} & b^{\prime \prime} \\ c^{\prime \prime} & d^{\prime \prime}\end{array}\right)$.

In the equivalence data, we take 
a) $\rho\left(C_{1}\right)=\left(\begin{array}{ll}1 & \frac{b}{d} \\ 0 & 1\end{array}\right), \quad \rho\left(C_{2}\right)=\left(\begin{array}{ll}1 & 0 \\ 0 & 1\end{array}\right), \quad \rho\left(C_{3}\right)=\left(\begin{array}{rr}1 & -\frac{b^{\prime}}{a^{\prime}} \\ 0 & 1\end{array}\right)$

or

b) $\rho\left(C_{1}\right)=\left(\begin{array}{rr}1 & -\frac{b}{a} \\ 0 & 1\end{array}\right), \quad \rho\left(C_{2}\right)=\left(\begin{array}{ll}1 & 0 \\ 0 & 1\end{array}\right), \quad \rho\left(C_{3}\right)=\left(\begin{array}{ll}1 & 0 \\ \frac{c^{\prime}}{a^{\prime}} & 1\end{array}\right)$

then

$$
\begin{aligned}
& P^{\prime}\left(C_{1}, C_{2}\right)=\rho\left(C_{1}\right)^{-1} P\left(C_{1}, C_{2}\right) \rho\left(C_{2}\right)=\left(\begin{array}{ll}
a & 0 \\
0 & d
\end{array}\right) \\
& P^{\prime}\left(C_{2}, C_{3}\right)=\rho\left(C_{2}\right)^{-1} P\left(C_{2}, C_{3}\right) \rho\left(C_{3}\right)=\left(\begin{array}{ll}
a^{\prime} & 0 \\
0 & d^{\prime}
\end{array}\right) .
\end{aligned}
$$

Consequently we get

$$
P^{\prime}\left(C_{3}, C_{1}\right)=\left(\begin{array}{cc}
a^{-1} a^{-1} & 0 \\
0 & d^{-1} d^{\prime-1}
\end{array}\right)
$$

from the relation

$$
P^{\prime}\left(C_{1}, C_{2}\right) P^{\prime}\left(C_{2}, C_{3}\right) P^{\prime}\left(C_{3}, C_{1}\right)=\left(\begin{array}{ll}
1 & 0 \\
0 & 1
\end{array}\right)
$$

So $E$ is decomposable thus we may assume that $\alpha_{i}$ are positive integers.

Case a,1) In the above argument, we may take $c^{\prime \prime}=0$. So the vector bundle $E$ is decomposable.

Case a,2) This case means that

$$
P\left(C_{1}, C_{2}\right)=\left(\begin{array}{ll}
a & b \\
0 & d
\end{array}\right), \quad P\left(C_{2}, C_{3}\right)=\left(\begin{array}{ll}
a^{\prime} & b^{\prime} \\
0 & d^{\prime}
\end{array}\right), \quad P\left(C_{3}, C_{1}\right)=\left(\begin{array}{cc}
a^{\prime \prime} & 0 \\
c^{\prime \prime} & d^{\prime \prime}
\end{array}\right) .
$$

From the relation

$$
P\left(C_{1}, C_{2}\right) P\left(C_{2}, C_{3}\right) P\left(C_{3}, C_{1}\right)=\left(\begin{array}{ll}
1 & 0 \\
0 & 1
\end{array}\right)
$$

we have

$$
c^{\prime \prime}=0 \text { and } a b^{\prime}+b d^{\prime}=0
$$

We take 


$$
\rho\left(C_{1}\right)=\rho\left(C_{3}\right)=\left(\begin{array}{ll}
1 & 0 \\
0 & 1
\end{array}\right), \quad \rho\left(C_{2}\right)=\left(\begin{array}{lr}
1 & -\frac{b}{a} \\
0 & 1
\end{array}\right)
$$

then

$$
\begin{aligned}
& P^{\prime}\left(C_{1}, C_{2}\right)=\rho\left(C_{1}\right)^{-1} P\left(C_{1}, C_{2}\right) \rho\left(C_{2}\right)=\left(\begin{array}{ll}
a & 0 \\
0 & b
\end{array}\right) \\
& P^{\prime}\left(C_{2}, C_{3}\right)=\rho\left(C_{2}\right)^{-1} P\left(C_{2}, C_{3}\right) \rho\left(C_{3}\right)=\left(\begin{array}{cc}
a^{\prime} & b^{\prime}+\frac{b}{a} d^{\prime} \\
0 & b^{\prime}
\end{array}\right)=\left(\begin{array}{ll}
a^{\prime} & 0 \\
0 & b^{\prime}
\end{array}\right) \\
& P^{\prime}\left(C_{3}, C_{1}\right)=\rho\left(C_{3}\right)^{-1} P\left(C_{3}, C_{1}\right) \rho\left(C_{1}\right)=\left(\begin{array}{cc}
a^{\prime \prime} & 0 \\
c^{\prime \prime} & d^{\prime \prime}
\end{array}\right)=\left(\begin{array}{cc}
a^{\prime \prime} & 0 \\
0 & d^{\prime \prime}
\end{array}\right)
\end{aligned}
$$

So $E$ is decomposable.

Case a,3) In this case

$$
P\left(C_{1}, C_{2}\right)=\left(\begin{array}{ll}
a & b \\
0 & d
\end{array}\right), \quad P\left(C_{2}, C_{3}\right)=\left(\begin{array}{ll}
a^{\prime} & b^{\prime} \\
0 & d^{\prime}
\end{array}\right), \quad P\left(C_{3}, C_{1}\right)=\left(\begin{array}{cc}
a^{\prime \prime} & b^{\prime \prime} \\
c^{\prime \prime} & 0
\end{array}\right)
$$

Then

$$
P\left(C_{1}, C_{2}\right) P\left(C_{2}, C_{3}\right) P\left(C_{3}, C_{1}\right)=\left(\begin{array}{cc}
a a^{\prime} a^{\prime \prime}+\left(a b^{\prime}+b d^{\prime}\right) c^{\prime \prime} & a a^{\prime} b^{\prime \prime} \\
d d^{\prime} c^{\prime \prime} & 0
\end{array}\right)
$$

This contradicts the relation

$$
P\left(C_{1}, C_{2}\right) P\left(C_{2}, C_{3}\right) P\left(C_{3}, C_{1}\right)=\left(\begin{array}{ll}
1 & 0 \\
0 & 1
\end{array}\right)
$$

So this case cannot happen.

Case a,4) This case cannot happen for the same reason as in the case a, 3).

Case $b, 1)$ This case determine the decomposable vector bundle for the same reason of the case a, 2).

Case $b, 2)$ This case cannot happen for the same reason of the case a, 3).

Case $b, 3)$ In this case

$$
P\left(C_{1}, C_{2}\right)=\left(\begin{array}{ll}
a & b \\
0 & d
\end{array}\right), \quad P\left(C_{2}, C_{3}\right)=\left(\begin{array}{ll}
a^{\prime} & 0 \\
c^{\prime} & d^{\prime}
\end{array}\right), \quad P\left(C_{3}, C_{1}\right)=\left(\begin{array}{cc}
a^{\prime \prime} & b^{\prime \prime} \\
c^{\prime \prime} & 0
\end{array}\right) .
$$

By taking 


$$
\rho\left(C_{1}\right)=\left(\begin{array}{ll}
a & 0 \\
0 & d
\end{array}\right), \quad \rho\left(C_{2}\right)=\left(\begin{array}{ll}
1 & 0 \\
0 & 1
\end{array}\right), \quad \rho\left(C_{3}\right)=\left(\begin{array}{cc}
a^{\prime-1} & 0 \\
0 & d^{\prime-1}
\end{array}\right)
$$

we may assume that

$$
a=d=a^{\prime}=d^{\prime}=1, \quad b^{\prime \prime} c^{\prime \prime}=-1 .
$$

Since

$$
P\left(C_{1}, C_{2}\right) P\left(C_{2}, C_{3}\right) P\left(C_{3}, C_{1}\right)=\left(\begin{array}{cc}
\left(1+b c^{\prime}\right) a^{\prime \prime}+b c^{\prime \prime} & \left(1+b c^{\prime}\right) b^{\prime \prime} \\
c^{\prime} a^{\prime \prime}+c^{\prime \prime} & c^{\prime} b^{\prime \prime}
\end{array}\right)
$$

we have

$$
\begin{gathered}
\left(1+b c^{\prime}\right) a^{\prime \prime}+b c^{\prime \prime}=c^{\prime} b^{\prime \prime}=1 \\
\left(1+b c^{\prime}\right) b^{\prime \prime}=c^{\prime} a^{\prime \prime}+c^{\prime \prime}=0 .
\end{gathered}
$$

Then

$$
b^{\prime \prime}=-b, \quad c^{\prime \prime}=b^{-1}, \quad c^{\prime}=-b^{-1}, \quad a^{\prime \prime}=1 .
$$

Furthermore we take

$$
\rho\left(C_{1}\right)=\rho\left(C_{2}\right)=\rho\left(C_{3}\right)=\left(\begin{array}{ll}
b & 0 \\
0 & 1
\end{array}\right),
$$

then we may assume that

$$
P\left(C_{1}, C_{2}\right)=\left(\begin{array}{ll}
1 & 1 \\
0 & 1
\end{array}\right), \quad P\left(C_{2}, C_{3}\right)=\left(\begin{array}{cc}
1 & 0 \\
-1 & 1
\end{array}\right), \quad P\left(C_{3}, C_{1}\right)=\left(\begin{array}{cc}
1 & -1 \\
1 & 0
\end{array}\right) .
$$

We note that transition matrices in the example are

$$
\begin{aligned}
& P\left(C_{2}, C_{1}\right)=P\left(C_{1}, C_{2}\right)^{-1}=\left(\begin{array}{cc}
1 & -1 \\
0 & 1
\end{array}\right) \\
& P\left(C_{3}, C_{2}\right)=P\left(C_{2}, C_{3}\right)^{-1}=\left(\begin{array}{ll}
1 & 0 \\
1 & 1
\end{array}\right) \\
& P\left(C_{1}, C_{3}\right)=P\left(C_{3}, C_{1}\right)^{-1}=\left(\begin{array}{cc}
0 & 1 \\
-1 & 1
\end{array}\right) .
\end{aligned}
$$

So this case determines the vector bundle of the type of the example.

Case b,4) For the same reason as in the case b,3), we may assume that

$$
P\left(C_{1}, C_{2}\right)=\left(\begin{array}{ll}
1 & b \\
0 & 1
\end{array}\right), \quad P\left(C_{2}, C_{3}\right)=\left(\begin{array}{ll}
1 & 0 \\
c^{\prime} & 1
\end{array}\right)
$$




$$
P\left(C_{3}, C_{1}\right)=\left(\begin{array}{cc}
0 & b^{\prime \prime} \\
c^{\prime \prime} & d^{\prime \prime}
\end{array}\right), \quad b^{\prime \prime} c^{\prime \prime}=-1
$$

Then

$$
P\left(C_{1}, C_{2}\right) P\left(C_{2}, C_{3}\right) P\left(C_{3}, C_{1}\right)=\left(\begin{array}{cc}
b c^{\prime \prime} & \left(1+b c^{\prime}\right) b^{\prime \prime}+b d^{\prime \prime} \\
c^{\prime \prime} & c b^{\prime \prime}+d^{\prime \prime}
\end{array}\right)
$$

So $c^{\prime \prime}$ must be zero, then $b c^{\prime \prime}=0$. This is a contradiction. This case cannot happen.

Thus every indecomposable vector bundle of rank 2 on $\boldsymbol{P}^{2}$ is of the form

$$
E(a, b, c) \otimes O_{P^{2}}(n)
$$

for some integer $n$ and some positive integers $a, b, c$.

Q.E.D.

Remark 5.2. If $a=b=c=1$, then the vector bundle $E(1,1,1) \otimes$ $O_{P_{2}}(n)$ is homogeneous, i.e. equivariant with respect to the standard action of $P G L(2)$ on $\boldsymbol{P}^{2}$. Conversely every homogeneous indecomposable vector bundle of rank 2 on $P^{2}$ is necessarily of the form $E(1,1,1) \otimes \underline{Q}_{P^{2}}(n)$ $(\operatorname{ch} k=0)$. [See 5]

The following problems can be posed about equivariant vector bundles on almost homogeneous varieties.

Problem 5.3. Classification of equivariant vector bundles of rank greater than 2 .

Problem 5.4. Is there any indecomposable equivariant vector bundle of $2 \leqq$ rank $\leqq n-1$ on $P^{n}(n \geqq 3)$ ? We can construct those of rank $n$ as in the case of 2 .

Problem 5.5. Classification of equivariant vector bundles on $X$, when $X$ is an almost homogeneous variety of dimension 2 .

\section{REFERENCES}

[1] Borel; Linear algebraic groups, Benjamin.

[2] G. Kempf et al.; Toroidal embeddings I, Springer lecture Notes 339.

[ 3 ] M. Maruyama; On a family of algebraic vector bundles, Number theory, Algebraic Geometry and commutative algebra, in honor of Y. Akizuki (1973), 95-146.

[4] T. Oda and K. Miyake; Almost homogeneous algebraic varieties under torus action, Manifolds Tokyo 1973, 373-381, Proceedings of the International Conference on 
Manifolds and Related Topics in Topology, University of Tokyo Press.

[5] M. Miyanishi; Some remarks on algebraic homogeneous vector bundles, Number theory, Algebraic Geometry and commutative algebra, in honor of Y. Akizuki (1973), 71-93.

[6] H. Sumihiro; Equivariant completion, Journal of Math. Kyoto Univ. 14 (1974), 1-28.

Nagoya University 